\title{
A City-Wide Vehicular Infrastructure for Wide-area Wireless Experimentation
}

\author{
Justin Ormont ${ }^{1}$, Jordan Walker ${ }^{1}$, Suman Banerjee ${ }^{1}$ \\ Ashwin Sridharan ${ }^{2}$, Mukund Seshadri ${ }^{2}$, Sridhar Machiraju ${ }^{2}$ \\ ${ }^{1}$ University of Wisconsin-Madison, WI, USA; ${ }^{2}$ Sprint Laboratories, Burlingame, CA, USA \\ Email: \{ormont, jwalker, suman\}@cs.wisc.edu, \\ \{ashwin.sridharan,mukund.seshadri,machiraju\}@sprint.com
}

\begin{abstract}
We describe our experiences in building a city-wide infrastructure for wide-area wireless experimentation. Our infrastructure has two components - (i) a vehicular testbed consisting of wireless nodes, each equipped with both cellular (EV-DO) and WiFi interfaces, and mounted on city buses plying in Madison, Wisconsin, and (ii) a software platform to utilize these testbed nodes to continuously monitor and characterize performance of large scale wireless networks, such as city-wide mesh networks, unplanned deployments of WiFi hotspots, and cellular networks. Beyond our initial efforts in building and deploying this infrastructure, we have also utilized it to gain some initial understanding of the diversity of user experience in large-scale wireless networks, especially under various mobility scenarios. Since our vehicle-mounted testbed nodes have fairly deterministic mobility patterns, they provide us with much needed performance data on parameters such as RF coverage and available bandwidth, as well as quantify the impact of mobility on performance. We use our initial measurements from this testbed to showcase its ability to provide an efficient, low-cost, and robust method to monitor our target wireless networks. These initial measurements also highlight the challenges we face as we continue to expand this infrastructure. We discuss what these challenges are and how we intend to address them.
\end{abstract}

\section{Categories and Subject Descriptors}

C.2.1 [Computer-communication networks]: Network Architecture and Design-Wireless communication; C.2.3 [Computer-communication networks]: Network Operations - Network monitoring

\section{General Terms \\ Design, Experimentation, Measurement, Performance}

Permission to make digital or hard copies of all or part of this work for personal or classroom use is granted without fee provided that copies are not made or distributed for profit or commercial advantage and that copies bear this notice and the full citation on the first page. To copy otherwise, to republish, to post on servers or to redistribute to lists, requires prior specific permission and/or a fee.

WinTech'08, September 19, 2008, San Francisco, California, USA.

Copyright 2008 ACM 978-1-60558-187-3/08/09 ...\$5.00.

\section{Keywords}

3G, Cellular, EVDO, Monitoring, Wide-area wireless, WiFi, Testbed, WiFi

\section{INTRODUCTION}

Wireless communication technologies are placing increasing emphasis on mobility and wide area coverage. A case in point is the EV-DO (EVolution-Data Only) 3G cellular network [10], that is widely deployed across the United States. This network provides high speed data connectivity over large geographic areas to both stationary and fast-moving mobile users. The peak data rates offered by this network are around 3 Mbps; however, in general, it is possible that the performance can vary based on location and time.

Conducting detailed performance measurements is a precursor to understanding, predicting, and debugging performance problems of any network. In a wired network, most measurement tasks involve deployment of a few monitoring nodes in carefully chosen vantage points within the network [3]. In a wireless environment, however, observations are a property of the physical location and its RF characteristics. In (relatively small) enterprise WLAN environments, it is sometimes feasible to deploy a moderate number of static wireless monitoring nodes to measure performance across the whole network, thereby creating a detailed view [14]. However, due to the geographic scale and diversity of a wide-area wireless network, such statically deployed monitoring nodes are mostly inadequate. In particular, such static monitors are unable to capture the impact of mobility on user performance. The goal of this paper is to investigate some effective strategies to monitor and measure performance across large-scale wide-area wireless networks.

Over the last ten months, we have been involved in a sustained effort to build a suitable infrastructure that meets this goal. This paper captures our initial measurement studies of utilizing this infrastructure that was conducted between March 17 and May 10, 2008.

Infrastructure overview: Our infrastructure for widearea wireless experimentation has two major components - a vehicular testbed that consists of wireless nodes, each equipped with both a cellular EV-DO and a WiFi interface and a GPS receiver, and a software platform to continuously monitor performance characteristics of the wide-area networks under test. Our (currently) dual-interface testbed wireless nodes are mounted on city buses plying in Madison, Wisconsin. Each city bus operates on multiple routes on a single day and is, therefore, able to traverse through 
significant parts of the city in each day. The bus routes are fairly deterministic. This allows for repeatable experimentation. Our starting deployment has been for two city buses. In the coming months, we expect to substantially augment this infrastructure to tens of buses, and equip each node with other wireless interfaces, including GNURadiobased SDR interfaces to facilitate experimentation in agile channel adaptation mechanisms for vehicular environments.

Since part of our goal is to understand the user-observable performance on these networks, we use end-user devices, such as laptops, as monitoring nodes. We also envision that, in the future, we will implement and deploy our continuous monitoring capabilities in other end-user devices, such as cellphones and PDAs, which are easier to deploy with regular users. (Challenges in meeting this objective are outlined in Section 6.) We call this approach, where client devices assist in network monitoring, client-assisted monitoring. We have developed an initial incarnation of a client-assisted monitoring platform as a software module, running on the vehiclemounted nodes.

Client-assisted network monitoring has many advantages. The popularization of powerful cellular-enabled devices offers us the opportunity to redefine the role of clients in this task. Cellular data interfaces are easily available for laptops, and modern handhelds (e.g., HTC Mogul) which have significant processing power and storage. This also allows us to shift the burden of network monitoring and data acquisition from a network-centric infrastructure to the end-nodes themselves. This reduces the prohibitive costs associated with network-based fine-grained monitoring infrastructure. Furthermore, the client devices are naturally mobile, and are therefore positioned perfectly to monitor performance in mobile scenarios and different locations.

In order to elicit the cooperation of the city bus system of Madison, we also implemented an Internet access service through our testbed nodes and made it available to the bus passengers. In particular, the testbed node also operates as a Mobile Access Router (MAR) - a device that serves as a WiFi Access Point to bus passengers and utilizes its 3G EV-DO interface to provide Internet services through it [11].

Our initial use of our testbed infrastructure is to measure the performance of a cellular EV-DO network. Our infrastructure highlights the benefits and challenges of such an effort and can potentially serve as a platform for various large-scale research activities requiring wide-area coverage and mobility support. This paper focuses on describing this testbed, the monitoring platform, and our future plans in this direction.

Summarizing, our wide-area wireless infrastructure possesses three main characteristics:

1. Data Acquisition: The basic element in our testbed is a dual interface equipped client node (a laptop with an EV-DO USB interface and a WiFi interface) running software that monitors and stores some desired characteristics pertaining to the wireless network. The cellular interface provides continuous remote access to each testbed node to experimenters. The client node uses it to periodically upload measurement data to a backend database system.

2. Location sensing: The client nodes are equipped with the capability to obtain current location from a GPS receiver. Thus, all acquired data is associated with the location where it was measured.

3. Mobility and Wide-area coverage: In our current prototype, the monitoring client devices are deployed on city buses plying in the city of Madison, Wisconsin. Thus, the testbed nodes are extremely mobile and cover a wide geographic area.

Uses of this testbed: Our testbed can be put into many different experimental uses. For example, the nodes can be utilized to collect information on WiFi deployment within the city scale [13]. They can also be used to test the performance of a city-wide WiFi-based wireless mesh network, available in the city of Madison. Given that the wireless nodes are mounted on multiple buses, they can also be utilized to understand characteristics of contact opportunities between these vehicles [15]. While we envision the multipurpose use of our testbed, our initial plans are to utilize this testbed to understand and troubleshoot performance issues of a large-scale EV-DO-based cellular network available in the Madison metropolitan area. The unique scale and reachability of this testbed is likely to provide interesting insights into the performance and characteristics of these networks.

At the current time, our testbed nodes are configured to monitor signal strength and conduct periodic measurements of a 3G cellular network. Our efforts are focused at characterizing the behavior of this network at scale, and conduct fine-grained analysis of this data.

Contributions: This paper captures the following key contributions:

First, our infrastructure highlights the challenges involved in implementing and deploying a vehicular infrastructure that operates in the scale of a city. We rig mechanisms to acquire continuous access to these nodes through a control channel, and devise strategies for remotely managing them. We also gain insights into various limitations of using such an infrastructure for the different granularities of measurement possible.

Second, we have collected a large volume of $3 \mathrm{G}$ cellular network measurements; at the time of this writing, more than 500 hours of mobile, and 350 hours of stationary measurements have been collected. These measurements can be analyzed to better understand performance. For example, we can draw coverage maps, analyze performance at specific locations, infer mobility patterns and study relationships between performance and mobility, or between performance and time.

Finally, through our experience we have been able to identify strategies by which we can significantly extend our testbed so that the monitoring and data acquisition functionality is deployed on a larger number of vehicle-mounted laptops as well as user-carried hand-held devices, in order to perform controlled wide-area measurements and data acquisition. Our next steps in this regard will to be to better understand the trade-offs between load (in terms of bandwidth and power, for example), monitoring accuracy and most importantly, power consumption. Our infrastructure provides a platform for experimentation and understanding of this trade-off.

The rest of this paper is organized as follows. Section 2 provides some background on the technologies and efforts involved in deploying the testbed. Section 3 describes the details of our testbed and measurement infrastructure. Sec- 
tion 4 presents results obtained from the initial measurements from our testbed. Section 5 surveys related work. We conclude in Section 6 with a summary, and a discussion of future work.

\section{BACKGROUND}

As discussed, the initial goal of our infrastructure is to facilitate easy collection of wide-area network performance data for a $3 \mathrm{G}$ EV-DO wireless network that would not otherwise be available. EV-DO is a high-speed, cellular data standard. The standardization process of EV-DO was undertaken by the Telecommunication Industry Association (TIA) as IS-856[10]. We provide a brief summary of the network below, highlighting salient details and refer the reader to the original specification document for more details. The EV-DO (Revision A) network has a peak download speed of $3.1 \mathrm{Mbps}$ and upload speed of $1.8 \mathrm{Mbps}$. The network is configured to provide wide-area coverage to stationary as well as highly mobile users through utilization of several communication technologies, such as adaptive rate realization, fast hand-off etc., .

The end-device performs rapid channel sensing in every slot (which is $1.667 \mathrm{~ms}$ ) to obtain an estimate of the received signal strength which is then used to request transmission at a specific data rate suitable for the particular channel conditions. The EV-DO network supports upto 12 distinct data rates. Based on this information, the basestation utilizes the Proportional Fair Scheduling algorithm [5] to decide on which user to serve in each slot, and then proceeds to transmit data at the specified data rate using an appropriate error coding rate and modulation that yields the desired rate. In order to avoid performance impairment due to mobility, the end-device also continuously monitors surrounding base-stations and as signal conditions change, it can request rapid hand-off from cell-tower to cell-tower.

A client-assisted monitoring platform with location sensing capabilities, such as ours, has many applications. This data gathered by this platform can assist in the following areas: (i) coverage mapping and compactly planning, (ii) hole detection, (iii) interference mitigation, (iv) channel planning and management, (v) wireless fault isolation and performance debugging, (vi) policy provisioning, and (vii) wireless security. We expect to build all of these capabilities as this project continues to evolve.

\section{TESTBED DESCRIPTION}

We now describe the design and implementation of our testbed infrastructure. As described, our initial goal of this deployment is to facilitate research in client-assisted widearea wireless network monitoring of cellular and other data networks.

Therefore, our testbed is based on client devices, equipped with both a cellular and a WiFi interface, running monitoring software, which periodically upload collected data to a back-end server. These client devices are deployed on Madison metro transit city buses. As a quid-pro-quo arrangement, we decided to provide wireless access to the bus passengers; this is accomplished using a MAR (Mobile Access Router) system - a system that serves as a WiFi Access Point to bus passengers, and hauls their wireless traffic to the Internet through the EV-DO interface [11]. We now describe each of these components in detail.

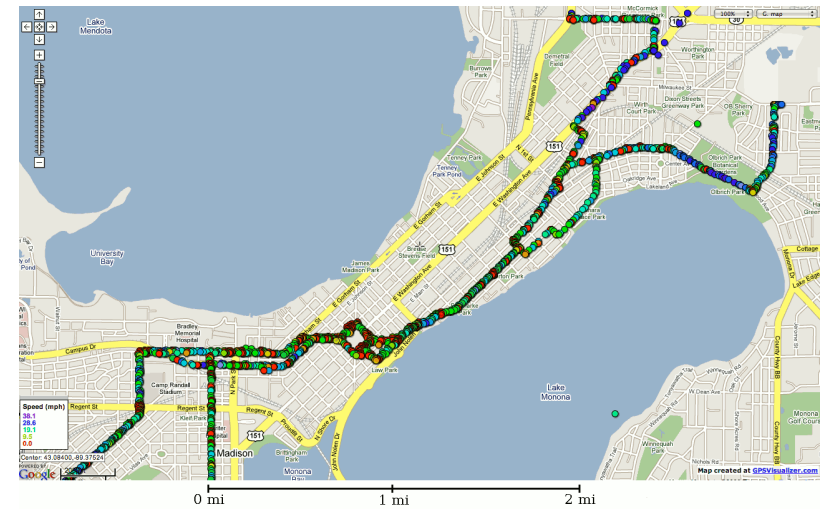

Figure 1: Trajectory of the city bus over a single representative day.

Vehicle: We require our testbed to cover a wide range of geographic areas. We achieve this target by deploying our monitoring client devices on a subset of buses in the Madison Metro (in Wisconsin, USA). While we plan to extend our deployment to multiple buses, we currently utilize two physical buses. However, these buses are not confined to any particular route. Together, these buses traverse several routes and cover a large fraction of the entire area of the city of Madison. Figure 1 shows the trajectory followed by one of these buses over the course of a typical day. This data was gathered using our monitoring infrastructure.

Client Device: The client device deployed on each Metro bus is the core of our testbed. It consists of a cellular-enabled laptop with an Intel Core 2 Duo 2.0 Ghz processor, 1 GByte of RAM, and a 120 GByte hard drive for storing collected data. For cellular connectivity the laptop uses one Sprint Novatel Wireless Ovation U727 1xEV-DO USB device. We plan to add additional EV-DO devices in the future. Additionally, we use a GlobalSat BU-353 USB NMEA receiver with the laptop, to enable GPS location tagging for all collected data.

MAR and additional functionality: In return for allowing us to deploy our devices, we provide wireless Internet access to the bus passengers. We achieve this by using a MAR system [11]. The MAR uses the idea that any technology that provides access to the larger network can be used to create a mobile access point for end-users. The MAR is used to provide wireless Internet service to the bus while we use the same equipment for our testbed.

In order to implement the MAR system, and for additional functionality, a Soekris Engineering Net4521 Linux integrated computer is used as an accessory to the platform. Other extensions may be added on to the Soekris computer; for example, we currently implement data collection regarding observed 802.11 channels (unrelated to the primary purpose of this paper). These extensions can then use the cellular interface to upload the data to a central location; this is facilitated by the MAR system.

Data Acquisition Modules: Our client devices have software and hardware modules or extensions that perform data acquisition tasks (for example, performance measurements) and store the results on the local hard drive. These logs are tagged with corresponding GPS coordinates and uploaded to a central location (the back-end server), where 
they are imported into a database. The GPS data from the GPS extension is crucial to the platform mobility, as the mobility is measured only through the GPS interface.

The network interface on which logs are uploaded is the EVDO cellular interface. Uploads are currently scheduled once every 2 minutes. This interface is also used for remote maintenance purposes: it allows for new extensions to be added, specific changes to be made, and problems to be corrected. Thus, the cellular interface has a role in all aspects of the platform; it acts as a tool for data collection, an interface for sending collected data, and a corridor for platform maintenance.

The extensions to the platform record data from one source and report it to the log system. No extension is required for the platform to operate, though some extensions may provide data upon which other extensions rely. For example, the mobility of the platform is contingent on the existence of a working GPS extension.

Any type of data can be collected through our extensions on the client device, and uploaded to the back-end server. In order to showcase the utility of a client-based wireless experimentation platform, we have chosen to focus on network performance monitoring as our initial focus of data acquisition for our testbed. This largely determines our initial choice of extensions to implement and deploy; we enumerate these extensions below.

1. GPS Location Extension: This extension provides the location information which gets associated with the other data. It searches the system for a suitable device, or multiple devices and polls for NMEA data. Redundant GPS devices are utilized for fail-over redundancy. When a GPS fix is achieved, it is parsed and logged to be later utilized when location tagging other data samples. In addition, studies are being done to determine the mode of transportation (e.g., walking, bus, etc.) based solely on GPS data gathered from device. A GPS location is recorded at the speed of the NMEA device (once per second).

2. Signal Strength Extension: Our testbed node utilizes a vendor-provided connection manager to connect to the cellular network. This program reports the signal strength in a report it generates. Polling this report every few seconds and recording it to file provides a picture of the signal strength variation over time and space. The mode of measurement intervals is once per 2 seconds.

3. ICMP Latency Extension: ICMP echo request packets are formed and sent through the Windows Management Interface (WMI) in sequence to a known IP address. The round-trip time is noted and logged. Loss rate is also measured through this method. The frequency of pings at a given time depends on the latency of the packets at said time and on average executes five times per second.

4. TCP 1MB Extension: The purpose of this extension is to initiate a TCP download and record the throughput achieved for the download. Currently we are downloading a $1 \mathrm{MB}$ file from our web server for this test. The duration of the download is used to determine the throughput, and after it completes another is initiated. The time associated with the throughput is the

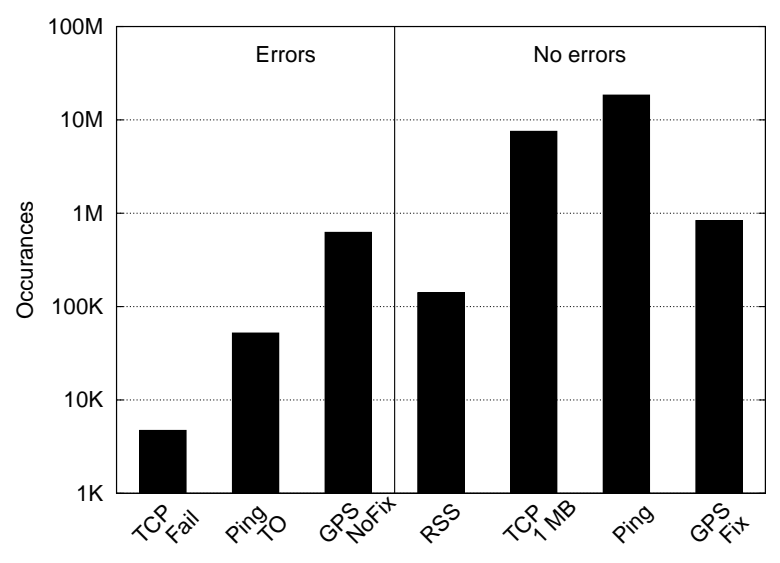

Figure 2: Number of recorded samples for each parameter currently tested. (Y-axis on log scale.) A small fraction of measurements returned errors.

completion time, so more data points occur when the throughput is higher, which must be considered when looking at the throughput data as a whole.

5. TCP 1 Sec Extension: A 1-second average of every network interface device is recorded. The incoming bitrate, outgoing bit-rate, incoming packets/second, and outgoing packets/second are recorded for each device. This provides us with a record of the bandwidth utilized by the MAR node which is later combined with data from the TCP $1 \mathrm{MB}$ Extension to provide a total bandwidth record. The TCP 1 Sec Extension can also be utilized to check the values for the TCP 1MB Extension and to provide a smaller granularity test of bandwidth. It executes once per second.

Back-end database: The back-end server employs a MySQL database set up to incorporate different data records with the mobility of the platform. Each record holds the time and GPS data associated with the data point as well as data to separate points of different collection nodes. A perl script parses the incoming logs into the database format as they are uploaded. This script is also responsible for correlating each point with GPS coordinates based on the closest GPS fix. The back-end also provides access so that applications can be built to utilize the data stored within the MySQL database.

\section{MEASUREMENT RESULTS}

This section provides an overview of the dynamics of the measurement testbed as well as some preliminary results obtained from the measurements for a city-wide $3 \mathrm{G}$ cellular data network. We present these results in order to demonstrate the feasibility and utility of the testbed, and to highlight challenges that arise and will need to be solved in the future, in a full-scale deployment.

In Figure 2, we present an overview of the number of samples we have collected through different measurements over a 1.5 month period. They include ping tests, TCP downloads, signal strength measurements, and GPS location information. The measurements are skewed based on time taken to collect each measurement (it takes much less time to collect 


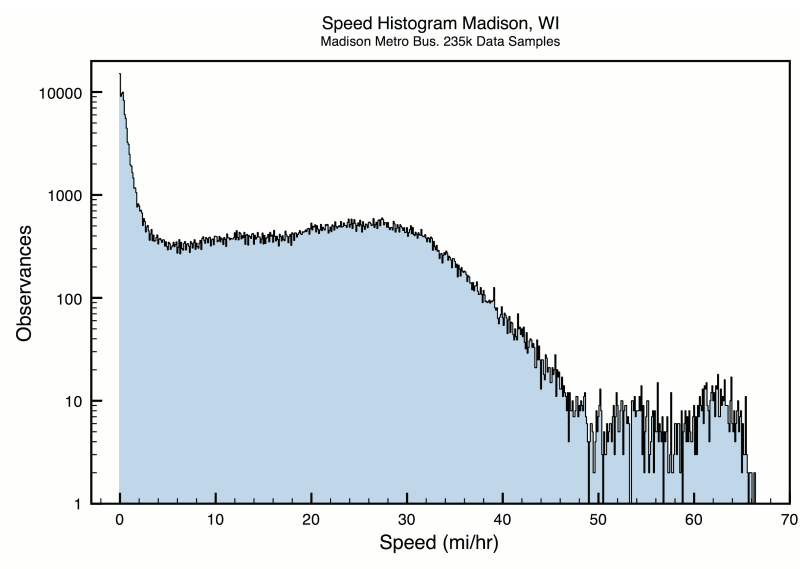

Figure 3: Distribution of vehicular speeds of one bus.

ping measurements than $1 \mathrm{MB}$ TCP downloads). The figure also shows that a small fraction of time, the ping and TCP experiments failed, as one can expect in any large scale experiment. Collecting GPS data was the most challenging component, and the number of failed GPS localization was almost the same as the number of successful fixes (possibly due to infrequent obscurity of the GPS satellites due to the position of the GPS receiver within the buses).

We now delve further into each measured variable, starting with the GPS location and speed information.

\subsection{Trajectory}

The measurement laptops were mounted on Madison Metro Buses which cover the entire city. Each bus operates on multiple routes on a given day. Hence, mounting nodes on even a few buses allows coverage of a large geographical region. Figure 1 shows the trajectory of one of these buses on a particular day, with "circular points" marking the position of this bus at different times of the day. The measurement also logs speeds which is represented by the shade of the circle.

A more detailed description of the speed of this bus is obtained via the associated density distribution plotted in Figure 3. The average speed of the bus was $14 \mathrm{mph}$. We observe from this figure that we obtain a large number of measurements for a variety of different speeds, up to $45 \mathrm{mph}$. Thus our testbed delivers useful observations of various levels of mobility.

The GPS receiver itself has some measurement errors that we needed to be cognizant of. In particular, we have observed that in stationary conditions, the GPS receiver indicated an average speed of $0.3 \mathrm{mph}$ with a variance of 1.3 mph (statistics of more than 15,000 samples).

\subsection{Network Performance Metrics}

We next turn to discussion of the relevant performance metrics obtained from our measurements. We focus on signal strength, latency (obtained through pings) and TCP throughputs, while taking location and mobility into consideration. While the buses were mobile most of the time they were active, they also had large durations of stationary situations, e.g., when parked at a transfer point, ready to start the next route.

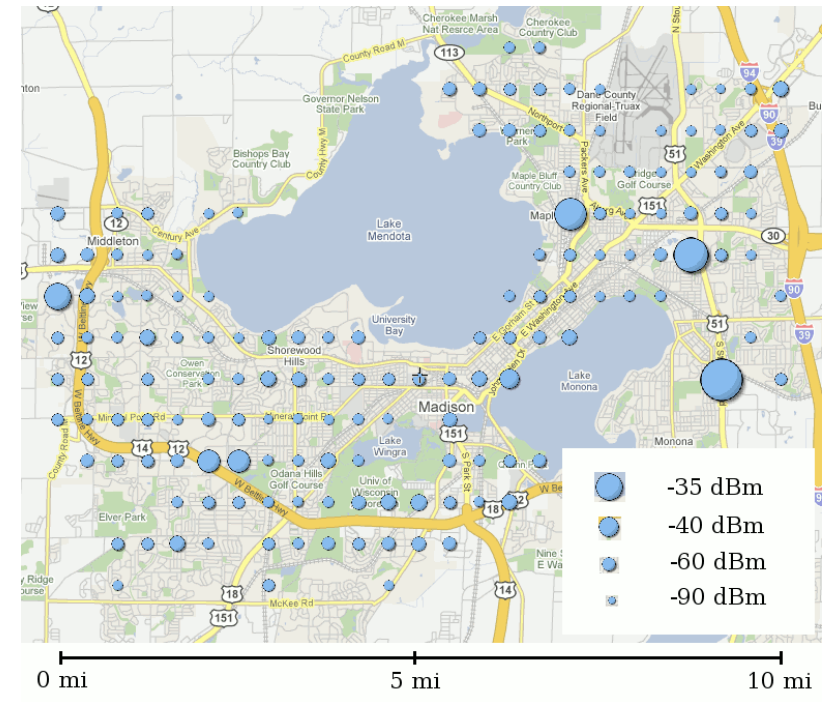

Figure 4: Signal strength map of Madison, WI.

\subsubsection{Signal Strength}

The basic metric in any wireless network is signal strength. Figure 4 shows a standard $R F$ coverage map obtained using our measurement infrastructure. The entire data set (consisting of more than 90,000 samples) was binned into a coarse granularity of 0.33 square miles for plotting convenience. Such measurements of coverage can be extremely useful for network planning and fault mitigation.

While mobile, the mean signal strength observed was $-77 \mathrm{dBm}$. When sampled from a specific location where the platform is known to be non-mobile, the mean signal strength was $-60 \mathrm{dBm}$. Clearly, there were a few locations with high signal quality, and many others with moderate signal quality.

While signal strength is a direct indicator of performance in wireless voice networks, this is not necessarily the case in data networks. We next look at two metrics which are more directly correlated with data performance of the network.

\subsubsection{Latency}

Figure 5 displays a city-wide latency map obtained via our measurement platform, illustrating latency as a function of location. Observe that across the entire geographical region, average latencies typically varied between 150 to $400 \mathrm{~ms}$.

\subsubsection{TCP throughput}

Finally, we present results on downlink throughput speeds obtained via TCP file download tests. In each test, 1 Mbyte file was downloaded from a known server. Second order effects due to potential caching by an intermediate proxy were removed by generating a random file name/content for each download.

Similar to the other two metrics, Figure 6 plots a coverage map highlighting network bandwidth as a function of location. We observe that the bandwidth map correlates well with the map of signal strength (Figure 4), indicating that better channel conditions typically lead to better download speeds. However, high user densities could lead to a reduction in bandwidth; thus a combination of these two maps could lead to a better understanding of user density and network load. This is an interesting future application of 


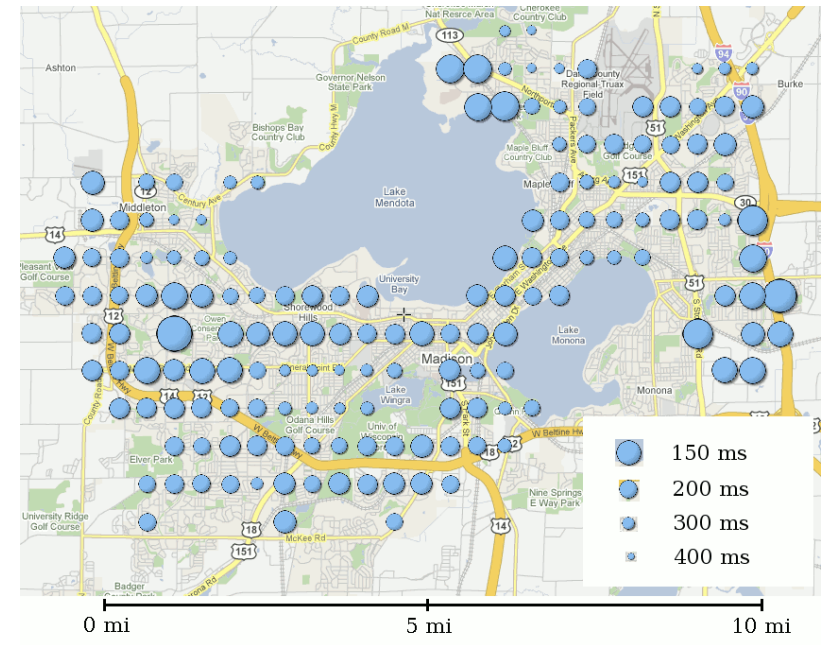

Figure 5: Latency distribution for a mobile client in Madison, WI.

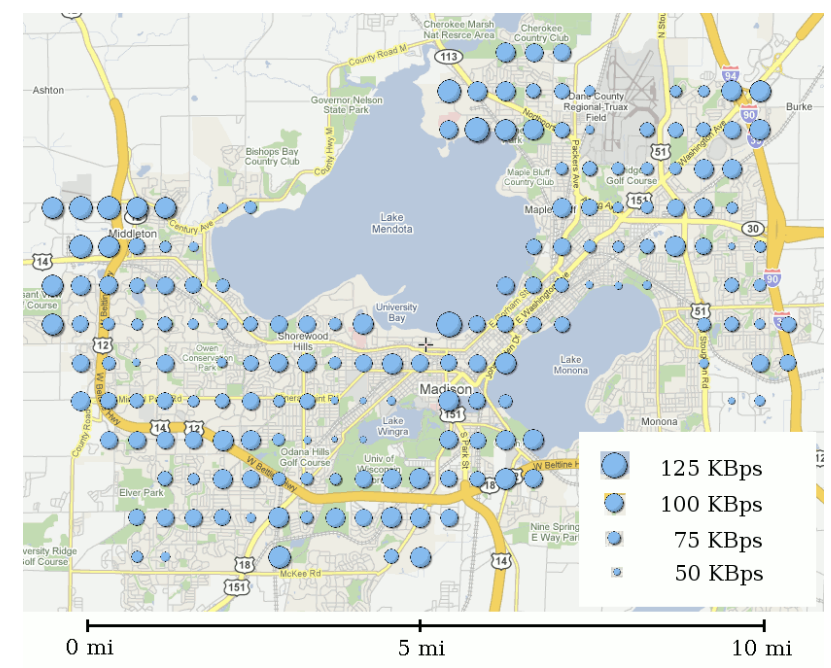

Figure 6: TCP throughput distribution with $1 \mathrm{MB}$ downloads in Madison, WI.

our measurements.

While mobile, the average sampled bandwidth was 94 KBps. While stationary, the average sample bandwidth was 100 KBps.

\subsubsection{Repeatability in a single stationary location}

So far, we have only described scenarios where the client device is mobile. However, there might be a need for measurements under stationary conditions too; for the sake of comparison; or to isolate temporal behavior at a single location. Our testbed possesses this functionality, to an extent. The buses stop in certain locations for long periods. We now present a result from such a scenario.

To demonstrate how latency varies over time at a single location, Figure 7 illustrates all observations at a "transfer point". Each bus, typically, waits an average of 20 minutes at this location prior to commencing its next route. These data points have been collected over multiple days. There

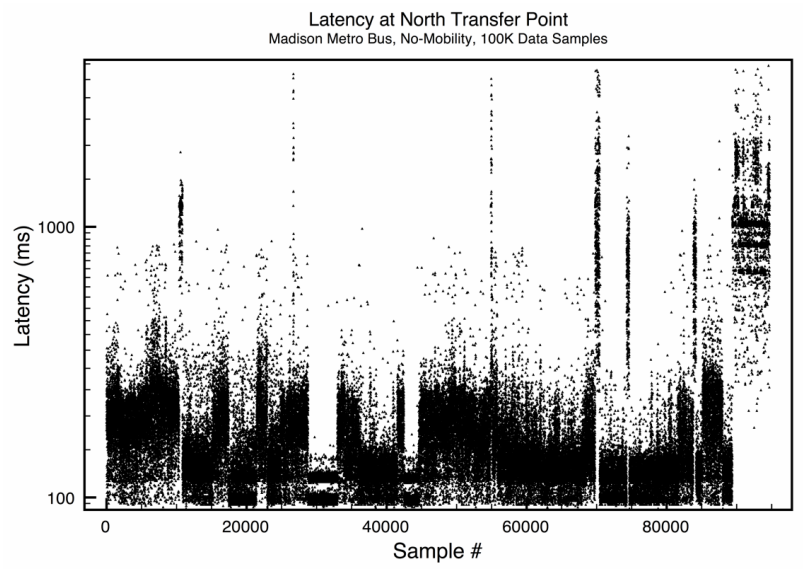

Figure 7: Distribution of latencies at the single most popular, stationary location, of the buses. (Y-axis on $\log$ scale.)

are expected variations of latency from this single location.

\section{RELATED WORK}

Performing large scale measurements in a wide-area mobile wireless environment is a constant challenge. To maintain realism of the environment, many logistical issues need to be solved. For example, one needs to provide an effective mechanism for continuous mobility. Similarly, it is important to facilitate remote management and experimentation for the mobile nodes. Remote management requires access to the nodes themselves, while experimentation requires the ease of downloading and installing new applications and systems on these nodes. Quite a few of our research efforts have had to deal with similar issues in the past, and in our current efforts, we have learned from all such prior efforts.

The earliest projects that involved significant mobile (vehicular) wireless testbeds for real-world experimentation were in the context of packet radio networks research [7] and DARPA's SURAN program [1]. Since then, multiple other DARPA efforts have utilized ad-hoc networking testbeds for various research activities.

One of the first efforts that use a 802.11-style wireless technology in evaluating perfomance of ad-hoc routing techniques, such as DSR [6], was the Monarch project at CMU through a vehicular testbed comprised of 5-6 car-mounted nodes [9]. The Kiosknet project at University of Waterloo, similarly, deployed 802.11 based wireless nodes in vehicles to provide DTN-based Internet services to remote villages in India [12].

A network of buses have been used in the DieselNet project, at UMass Amherst, to understand challenges and design considerations in general delay tolerant networks and in design of opportunistic communication [15]. Finally, the CarTel project at MIT uses a similar WiFi-based nodes and additional sensors on a fleet of 27 limousines to create real-time maps of road conditions and identify availability of various open (and closed) 802.11 Access Points visible in a city-wide scale $[4,13]$.

Our testbed deployment is uniquely different from these testbeds in multiple ways. First, one of the goals of our testbed is to serve as a platform for wide-area client-assisted 
wireless monitoring of large-scale wireless networks. Second, we utilize a cellular EV-DO based interface to provide a control channel back to the testbed nodes (not deployed in any of the other testbeds). Third, we also provide Internet access service through the testbed nodes, to bus passengers, thereby enabling a symbiotic relationship between this project and the bus operators. Finally, our future plans, of augmenting the testbed with SDR-based interfaces on vehicular nodes, and inducting cellular phones and PDAs for client-assisted monitoring, will significantly enhance desired capabilities.

There have been relatively few measurement based performanceevaluation studies on the 1xEV-DO network. Other research efforts $[8,2]$ have conducted transport layer measurements to infer TCP throughput and delays. However, these were conducted at static locations and did not cover mobility or multiple locations, which is a key feature of the client platform.

\section{CONCLUSIONS AND FUTURE WORK}

We presented our initial experiences with designing and deploying a wide-area testbed to monitor wide-area wireless networks. A key feature of our testbed is its reliance on a unique set of clients - laptops mounted on metro buses in the city of Madison equipped with both cellular and WiFi wireless cards - to continuously monitor and report data back to a centralized location. Metro buses provide us with three unique advantages. First, by providing coverage over relatively large portions of the city over a period of many days, they help us achieve scale quickly. Second, by piggybacking on metro buses, we are able to collect a large number of measurements at very little cost and effort. Third, the repeated traversal of a few routes by the buses help us conduct repeatable measurements.

Within the current testbed, we want to add greater capabilities to the wireless nodes mounted on each bus. Currently each testbed node has a EV-DO and a WiFi $(802.11 \mathrm{a} / \mathrm{b} / \mathrm{g})$ interface. We plan to augment them with an SDR interface, that will allow for software-level adaptations of the wireless channel in the highly mobile vehicular environments. Next, we intend to scale the size of this bus-mounted testbed. Madison's metro bus system operates more than 200 buses plying on different routes on a daily basis, and our long term goal is to expand the testbed to such a large scale.

We also plan to expand this testbed to operate on other platforms, including handheld devices, such as cellular phones. This will allow us to collect interesting measurement data from devices regular carried by cellular users. However, as we move from laptops powered by buses to battery-powered cellphones, we will face problems related to the resource limitations of clients. Additionally, as the number of measurement sources grow, we expect to face challenges related to the amount of data collected. We intend to pursue sampling strategies especially for the spatial dimension, as a way to achieve accurate results with minimal cost. Therefore, we plan to investigate how handheld device constraints, such as power and bandwidth, can be incorporated into our data collection mechanisms. As we continue to develop better mechanisms, we also intend to continue our data collection process and increase our understanding of large-scale network performance.

Finally, since our testbed involves a MAR system that provides Internet services to bus passengers, we will be in a unique position to monitor, understand, and characterize user behavior in this mobile environment. The users of MAR will also be sources of real user traffic passing through our monitoring nodes and will obviate the need to create artificial traffic on the system to understand performance.

\section{ACKNOWLEDGMENTS}

This work is supported in part by the US National Science Foundation under grants CNS-0639434, CNS-0627589, CNS0627102, CNS-0520152, and CNS-0747177.

\section{REFERENCES}

[1] D. Beyer. Accomplishments of the DARPA survivable adaptive networks SURAN program. In Milcom, 1990.

[2] M. Claypool, R. Kinicki, W. Lee, M. Li, and G. Ratner. Characterization by measurement of a CDMA 1xEVDO network. In Proc. of Wireless Internet, 2006.

[3] C. Fraleigh, S. Moon, B. Lyles, C. Cotton, M. Khan, D. Moll, R. Rockell, T. Seely, and C. Diot. Packet-level traÃEc measurements from the sprint IP backbone. In IEEE Network, 2003.

[4] B. Hull, V. Bychkovsky, K. Chen, M. Goraczko, A. Miu, E. Shih, Y. Zhang, H. Balakrishnan, and S. Madden. CarTel: A distributed mobile sensor computing system. In ACM Sensys, 2006.

[5] A. Jalai, R. Padovani, and R. Pankaj. Data Throughput of CDMA-HDR : A High Efficiency-high Data Rate Personal Communication Wireless System. In Proc. of IEEE Vehicular Technology Conference, May 2000.

[6] D. Johnson and D. Maltz. Dynamic Source Routing in Ad Hoc Wireless Networks, chapter 5, pages 153-181. Mobile Computing, edited by by Tomasz Imielinski and Hank Korth. Kluwer Academic Publishers, 1996.

[7] R. Kahn, S. Gronemeyer, J. Burchfiel, and R. Kunzelman. Advances in packet radio technology. IEEE, 66(11), Nov. 1978.

[8] Y. Lee. Measured TCP Performance in CDMA 1xEV-DO Network. In Proc. of Passive Active Measurement Conference, 2006.

[9] D. Maltz, J. Broch, and D. Johnson. Quantitative lessons from a full-scale multi-hop wireless ad hoc network testbed. In $W C N C, 2000$.

[10] QualComm. 1xEV: 1x EVolution, IS-856 TIA/EIA Standard. Available from www.3gpp2.org.

[11] P. Rodriguez, R. Chakravorty, J. Chesterfield, I. Pratt, and S. Banerjee. MAR: A commuter router infrastructure for the mobile internet. In $A C M$ Mobisys, 2004.

[12] A. Seth, D. Kroeker, M. Zaharia, S. Guo, and S. Keshav. Low-cost communication for rural internet kiosks using mechanical backhaul. In ACM Mobicom, Sept. 2006.

[13] B. V., B. Hull, A. Miu, H. Balakrishnan, and S. Madden. A measurement study of vehicular internet access using in situ Wi-Fi networks. In $A C M$ Mobicom, 2006.

[14] J. Yeo, M. Youssef, and A. Agrawala. A framework for wireless lan monitoring and its applications. In $A C M$ WiSe, 2004. 
[15] X. Zhang, J. Kurose, B. Levine, D. Towsley, and H. Zhang. Study of a bus-based disruption-tolerant network: Mobility modeling and impact on routing. In ACM Mobicom, Sept. 2007. 